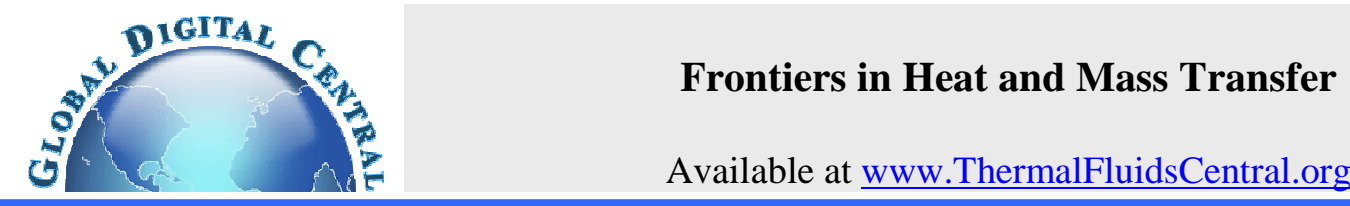

\title{
MODELLING OF COMBINED HEAT AND MASS TRANSFER OF WATER DROPLETS IN THERMAL TECHNOLOGY EQUIPMENT
}

\author{
Gintautas Miliauskas*, Stasys Sinkunas, Kristina Norvaisiene, Kestutis Sinkunas \\ Department of Thermal and Nuclear Energy, Kaunas University of Technology, K. Donelaicio 20, LT-44239, Kaunas, Lithuania
}

\begin{abstract}
Water droplet evaporation process is numerically modelled under various heat and mass transfer conditions. Regularities of heat transfer process interaction are examined. Modelling in this work was performed using the combined analytical - numerical method to investigate heat and mass transfer in the two-phase droplets-gas flow system. The influence of forced liquid circulation on the thermal state of droplets is taken into account by the effective coefficient of thermal conductivity. Calculating the rate of droplet evaporation and the intensity of convective heating, the influence of the Stefan's hydrodynamic flow is taken into account. Balancing energy fluxes in the droplet to within one hundredth of a percent and using the fastest sinking the droplet surface temperature is determined.
\end{abstract}

Keywords: water droplets, combined heating, evaporation.

\section{INTRODUCTION}

Heat and mass transfer in water droplets is important in many thermodynamic applications: air drying and humidification in evaporation chambers of HVAC systems, cooling of high temperature gas flows, fire extinguishing systems, etc. Water injection into combustion chamber or air supply system of internal combustion engine is an effective method to regulate combustion temperature or minimize $\mathrm{NO}_{\mathrm{x}}$ emission of combustion process. Scientists and industry are interest in water injection effect on compression and expansion processes in gas turbines. Aforementioned applications and processes are governed or influenced by water droplet evaporation. The rate of droplet heating is essential to droplet evaporation process in the twophase droplet-gas system.

In the evaporation chamber there is a small energy potential difference between air and water injected into it. Therefore, the process of phase transformation is inefficient. It is possible to intensify heat and mass transfer by delivery of additional heat into water droplets. This requires one to understand the influence of radiation to heat and mass transfer process of sprayed water droplets. It is necessary to take into account the intensity of heat and mass transfer in the air flow and inside the evaporating droplets. Processes of dispersion and two-phase flow are closely related (e.g., Miliauskas and Sabanas, 2006; Tseng and Viskanta, 2006). Research of heat and mass transfer of the sprayed liquid is known as "droplet problem". It covers research of heat and mass transfer phenomena inside the droplet and in its ambience. Last decade achievements in "droplet problem" are described in literature (e.g., Sazhin, 2006; Miliauskas et al., 2010).

\section{ANALYTICAL-NUMERICAL METHOD OF RESEARCH}

The combined analytical-numerical method to solve droplet problem is applicable when it is necessary to consider the interaction of unsteady heat and mass transfer process with the peculiarities of spectral radiation (Miliauskas, 2001). The advantages of this method include prevention of timing and space coordinate indeterminacies, as well as, reliable control of convergence. The method of droplet surface temperature calculation is based on the principle of energy flow balance at the droplet surface (see Fig. 1).

$$
\vec{q}_{\Sigma}^{+}(\tau)+\vec{q}_{\Sigma}^{-}(\tau)+\vec{q}_{f}^{+}(\tau)=0
$$

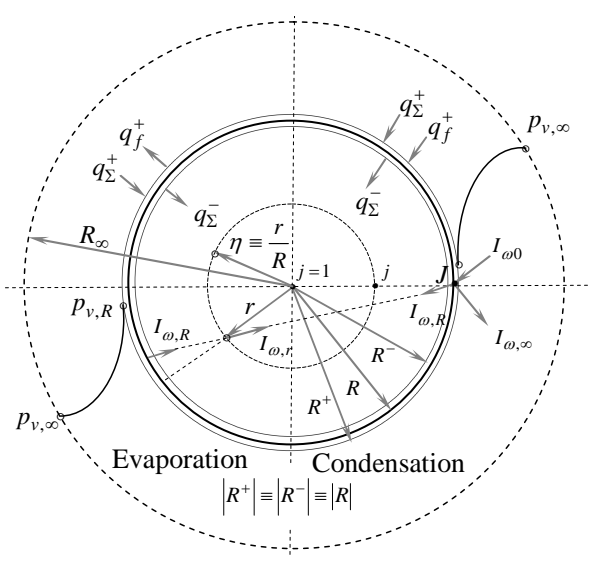

Fig. 1 Energy fluxes on the droplet surface.

Since the spectral radiation absorption coefficient of the semitransparent droplet are finite, radiation is not absorbed in a droplet surface: $q_{r}^{-}\left(R^{-}, \tau\right) \equiv q_{r}^{+}\left(R^{+}, \tau\right)$. Then the Eq. (1) can be rearranged.

$q_{c}^{+}(\tau)-q_{c}^{-}(\tau)-q_{f}^{+}(\tau)=0$

The external convective flow between gas and the droplet is

$q_{c}^{+}=\frac{N u \cdot \lambda_{v g}}{2 R} \cdot\left(T_{g}-T_{R}\right) \cdot f\left(B_{T}\right)$ 
The function of Spalding transfer number evaluates the influence of hydrodynamic Stephan flow. Nusselt number for the droplet is described by Abramzon-Sirignano model (e.g., Abramzon and Sirignano, 1989), and Spalding transfer number is determined based on recommendations presented in (e.g., Miliauskas and Sabanas, 2006).

The internal convective flow in a droplet is described by the modified Fourier law.

$$
q_{c}^{-}(\tau)=\left.\lambda_{e f}(\tau) \cdot \frac{\partial T(r, \tau)}{\partial r}\right|_{r \equiv R^{-}}
$$

The effective coefficient of thermal conductivity $\lambda_{e f} \equiv \lambda_{l} k_{c}$, which takes into account water circulation inside the droplet according to the recommendations in (e.g., Abramzon and Sirignano, 1989).

$k_{c}(\tau)=1.86+0.86 \tanh \left[2.245 \log _{10} \frac{P e_{l}(\tau)}{30}\right]$

Unsteady temperature field in water droplet is described by model of combined heat transfer in a droplet by conduction and radiation.

$$
\begin{aligned}
& T(r, t)=T_{R}(t)+\frac{2}{r} \sum_{n=1}^{\infty} \sin (n \pi \eta) \\
& \times \int_{0}^{t} f_{n}(\tau) \exp \left[-a\left(\frac{n \pi}{R}\right)^{2}(t-\tau)\right] d \tau
\end{aligned}
$$

Function $f_{n}$ takes into account the influence of radiation absorption in the droplet and the rate of change of surface temperature to the temperature field in the droplet (Miliauskas, 2001).

$$
\begin{aligned}
& f_{n}(\tau)=(1)^{n} \frac{R(\tau)}{n \pi} \frac{d T_{R}(\tau)}{d \tau}+\frac{1}{R(\tau) \rho(\tau) c_{p}(\tau)} \\
& \times \int_{0}^{R} q_{r}(r, \tau)[\sin (n \pi \eta)-n \pi \eta \cdot \cos (n \pi \eta)] d r
\end{aligned}
$$

Non-stationary temperature gradient in Eq. (4) is described by the following Eq. (8).

$\left.\frac{\partial T_{r}}{\partial r}\right|_{r \equiv R^{-}}=\frac{2 \pi}{R^{2}} \sum_{n=1}^{\infty} n(-1)^{n} \int_{0}^{t} f_{n}(\tau) \exp \left[-a\left(\frac{n \pi}{R}\right)^{2}(t-\tau)\right] d \tau$

The density of phase transformations heat flow

$q_{f}^{+}(\tau)=m_{v}^{+}(\tau) \cdot L(\tau)$

is determined by the density of vapor flow on droplet surface

$m_{v}^{+}(\tau)=\frac{D_{v g}(\tau) \mu_{v}}{T_{R, v g}(\tau) R_{\mu} R(\tau)} p \ln \frac{p-p_{v, \infty}(\tau)}{p-p_{v, R}(\tau)}$,

which evaluates the influence of Stefan flow to mass transfer (e.g., Shorin, 1964; Kuzikovskij, 1973). To determine the volume variation of a spherically symmetrical droplet the intensity of phase transformation on droplet surface and expansion of warming water are evaluated.

$\rho(\tau) \frac{\partial R^{3}(\tau)}{\partial \tau}=R^{3}(\tau) \frac{\partial \rho(\tau)}{\partial \tau}-3 R^{2}(\tau) m_{v}^{+}(\tau)$

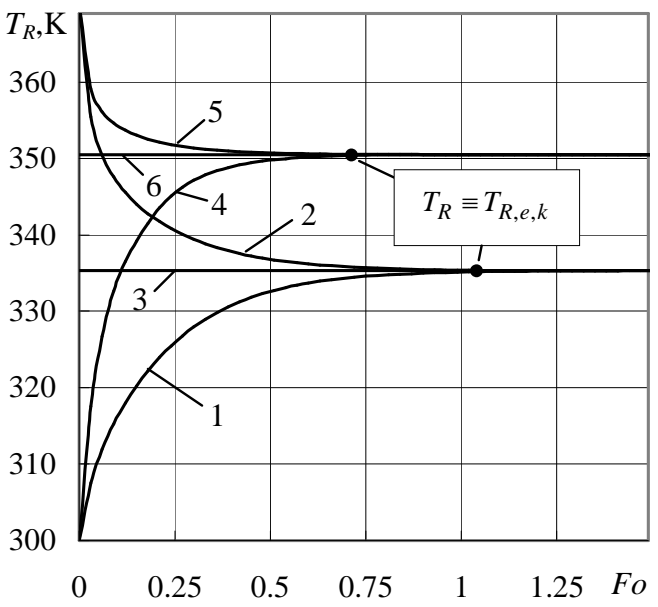

(a) Conductive heating

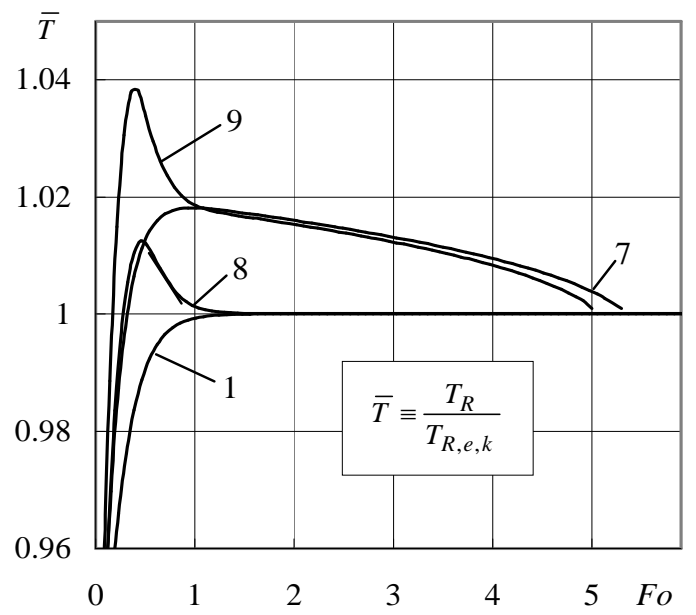

(b) Combined heating

Fig. 2 Droplet surface temperature dependence on heating manner: (16) conductive; (7) radiative-conductive; (8) convective; (9) radiative-convective. $T_{g}=1073 \mathrm{~K} . R_{0}=0,0002 \mathrm{~m}$. Droplet primary temperature $T_{l, 0}, \mathrm{~K}:(1,4,7-9) 300,(2,5) 370$, $(3,6) T_{R, e, k}$. Water vapour volumetric part in air $p_{v, \infty} / p:(1-3,7-9) 0,(4-6)$ 0.25 . Primary velocity of the droplets slip in air $\Delta w, \mathrm{~m} / \mathrm{s}:(1-7)$ $0,(8,9) 10$.

The system of Eqs.(2-11) is solved numerically using an iterative method. Number $J$ of a control droplet cross-section is freely selected. The position of cross-section is defined by dimensionless droplet coordinate $\eta: \eta_{j}=0$ when $j \equiv 1 ; \eta_{j}=1$ when $j \equiv J$. Control time $t$ is selected and number $I$ of time coordinate steps is provided: $\tau=0$ when $i \equiv 1 ; \quad \tau=t$ when $i \equiv I$. The thermal state of water droplet convectively heated by gas and influenced by radiation is calculated numerically solving the Eqn. (6). The warming droplet surface temperature is calculated using the method of steepest descent down to the accuracy of balance (2) being no less than one hundredth of the percent. The local radiant flux density in the water droplet is calculated by technique described in (Miliauskas, 2001) based on the temperature field calculated in previous iteration. The technique takes into account the radiation absorption inside the droplet and spectral effects on the surface of the contact between air and water. The spectral optical characteristics of water have been investigated very well (e.g., Hale and Querry, 1973; Hale et al., 1972). 


\section{RESULTS AND DISCUSSION}

The change of thermal state of waters, evaporating in air flow is modelled numerically under different conditions of heat and mass transfer, when liquid spray dispersity influence on gas flow parameters is insignificant. Conducting heating is modelled considering that the droplets are moving in air flow without slipping. In the case of convective heating, the initial velocity of the droplets slip in air is predicted. When considering combined heating of droplets, it is stated that absolutely black source of radiation exits, which temperature corresponds to the temperature of air flow.

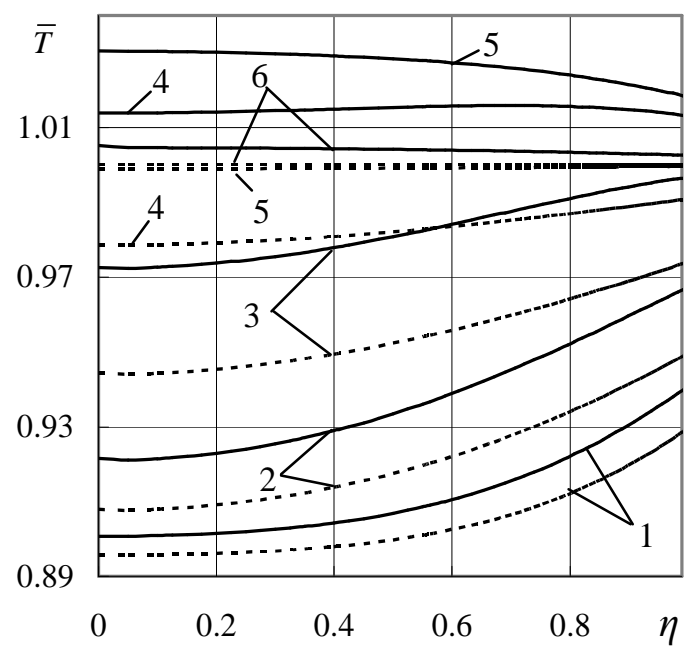

(a) "Cold" liquid

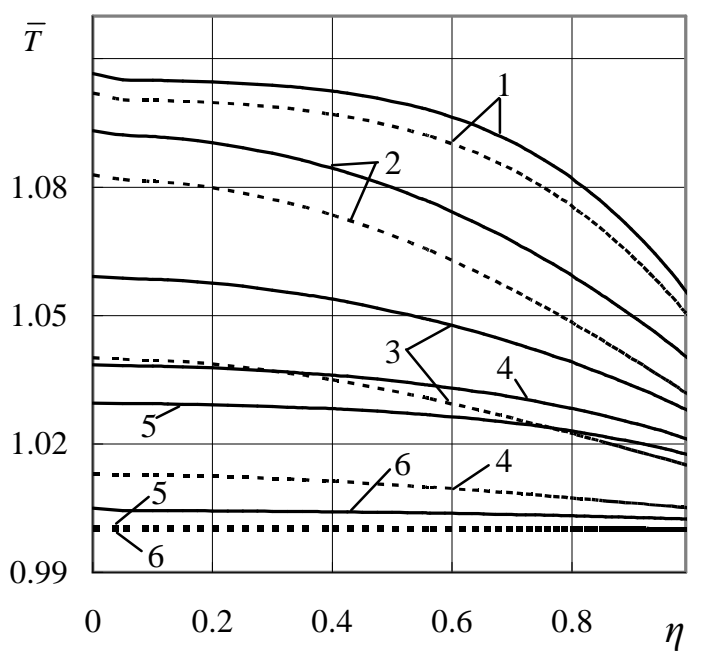

(b) "Hot" liquid

Fig. 3 Droplet temperature dependence on initial water temperature and droplet heating manner: (dotted lines) conductive heating, (solid lines) radiative - conductive heating. Initial water temperature $T_{l, 0}, \mathrm{~K}$ : (a) 300, (b) 370. Heating time $F_{o}$ : (1) 0.072 , (2) 0.144 , (3) 0.289 ; (4) 0.506 , (5) 1.08 , (6) 5.2. $T_{g}=1073 \mathrm{~K} . p=0.1 \mathrm{MPa} . R_{0}=0,0002 \mathrm{~m} . \bar{T} \equiv T(F o, \eta) / T_{R, e . k}$.

Comprehensive analysis of water droplet warming and evaporation results obtained under different boundary conditions of heat and mass transfer, highlighted the determinant influence of the water droplet heating manner on it state change dynamics (see Fig. 2).

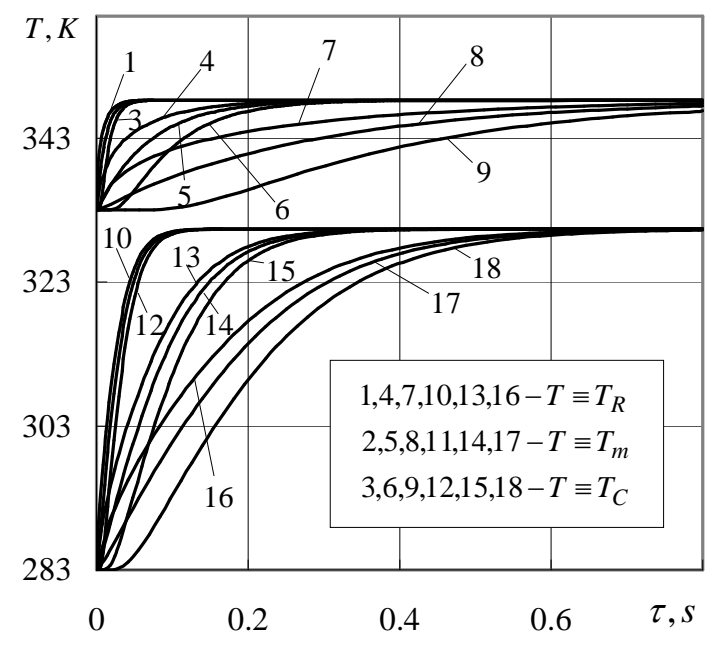

(a) Real time

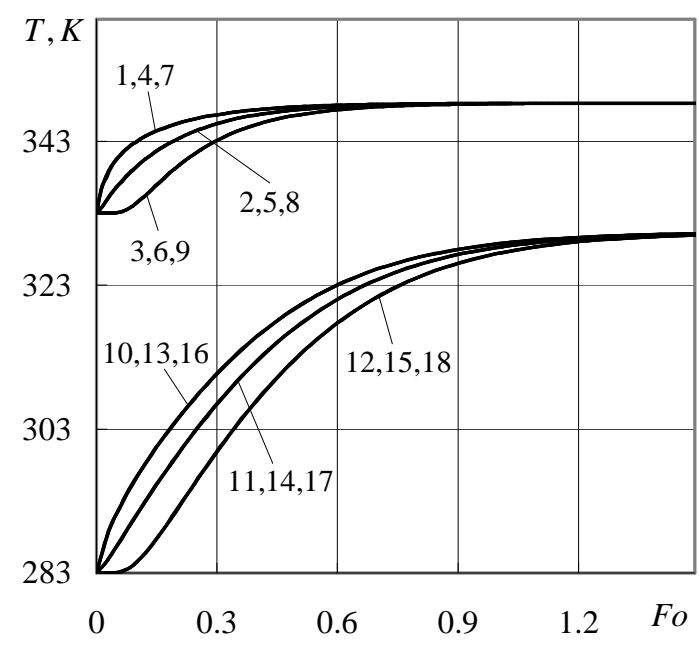

(b) Fourier number scale

Fig. 4 Dynamics of water droplet warming in a real time (a) and in a time scale expressed by Fourier number (b) in the case of conductive heating. $T_{g}=818 \mathrm{~K} . R_{0}$, m: $(1-3,10-12) 0.0001$, (46,13-15) 0.000175, (7-9,16-18) 0.00025. $p_{v, \infty} / p:(1-9) 0.25$, (10-18) 0 .

It was defined that transferring the heat from a gas to a droplet in different manners, independently on the same primary conditions, the droplet reaches equilibrium evaporation state being in different energy level. It is outlined by the droplet surface temperature at the beginning of equilibrium evaporation. Indication of the equilibrium evaporation all energy supplied to a droplet evaporates it: $q_{f}^{+} \equiv q_{\Sigma}^{+}$. It was noted that during equilibrium evaporation droplet surface temperature, independently on the heating manner, approaches certain distinctive temperature $T_{R, e, k}$, it corresponds to the droplet equilibrium evaporation temperature in conductive heating case (see Fig. 2). It was evaluated that this temperature is a function of the droplet surrounding gas temperature and liquid vapour partial pressure in it $T_{R, e, k}=f\left(T_{g}, p_{v, \infty}\right)$. It does not depend on the droplet primary state, it is the lowest droplet surface temperature at which droplet equilibrium evaporation at given $T_{g}$ and $p_{v, \infty}$ is possible. The lowest values temperature $T_{R, e, k}$ achieves when a droplet evaporates in a gas where there is no liquid vapour: when $p_{v, \infty} \equiv 0$, then $T_{R, e, k}=f\left(T_{g}\right)$. The value of the ratio of the initial liquid temperature $T_{l, 0}$ on temperature $T_{R, e}$ of liquid droplet that evaporates in equilibrium mode influences 
the change of the thermal state of sprayed liquid during unsteady phase transformations (e.g., Miliauskas and Garmus, 2004). As $T_{l, 0} / T_{R, e}<1$, droplets warm during unsteady phase transformations and it is possible to state that sprayed liquid is "cold". As $T_{l, 0} / T_{R, e}>1$, droplets cool during unsteady phase transformations and it is possible to state that sprayed liquid is "hot". As the value of temperature ratio $T_{l, 0} / T_{R, e}$ is close to 1 , the influence of unsteady phase transformation mode is negligible and the thermal state of the droplets changes insignificantly during this mode. The flow of radiation, absorbed in water, accelerates warming of cold water droplets (see Fig. 3a), but retards cooling of hot water droplets (see Fig. 3 b).

The change of characteristic centre, surface and mean mass temperatures of the unsteady temperature field is distinctive for water droplets of different size (Fig. 4 a). Though variation of the curves in Fourier number based scale is independent of droplet size (Fig. 4 b). Hence, in Fourier number based scale the characteristic curves indicate warming dynamics of infinite set of water droplets. This important conclusion allowed optimizing the whole numerical research (Miliauskas et al., 2008).

The imbalance of energy fluxes at the surface of evaporating liquids is controlled during the numerical experiment (see Fig. 5). In the selection of an instant value of the droplet surface temperature using the fastest descent method, the temperature variation in the range of onethousandth of a degree was considered. Then, the balance of energy fluxes is secured at a confidence not less than $0.05 \%$ during the numerical investigation. Imbalance fluctuation is related to the change of the phase transformation mode, when the vapor flux density module reaches its zero value and the direction of its vector changes as vapor condensation at the droplet surface is replaced by liquid evaporation (see Fig. 5). The control of the energy flux imbalance at the droplet surface was performed in all cases of phase transformations.

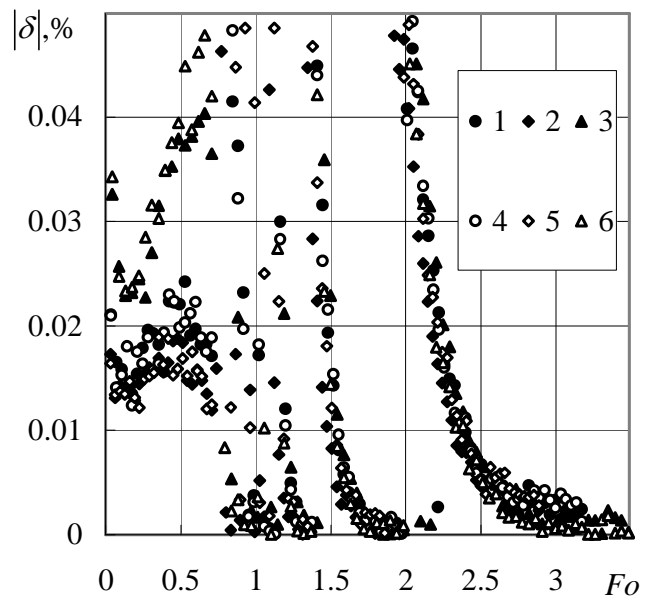

Fig. 5 Imbalance control of energy fluxes at the droplet surface during the numerical investigation. Droplet heating mode: (1-3) conductive, (4-6) radiative-conductive. $T_{l, 0}=278 \mathrm{~K} . R_{0}, \mathrm{~m}:(1$,

4) $0.000025,(2,5) 0.00005,(3,6) 0,0001 . T_{g}=500 \mathrm{~K}$. $p_{v, \infty} / p=0.1$.

State change of cold evaporating droplet may be classified by instantaneous temperature field peak position in the droplet, accentuating primary, transitive and final droplet heating periods. Zero value non-dimensional temperatures

$T_{n}(\eta, F o)=\frac{T(\eta, F o)-T_{\min }(\eta, F o)}{T \max (\eta, F o)-T_{\min }(\eta, F o)}$
$T_{n} \equiv 0$ shows minimal temperature position of instantaneous temperature field in a droplet (see Fig. 6) (Miliauskas, 2001). The value $T_{n} \equiv 1$ shows maximal temperature place of this field in a droplet.

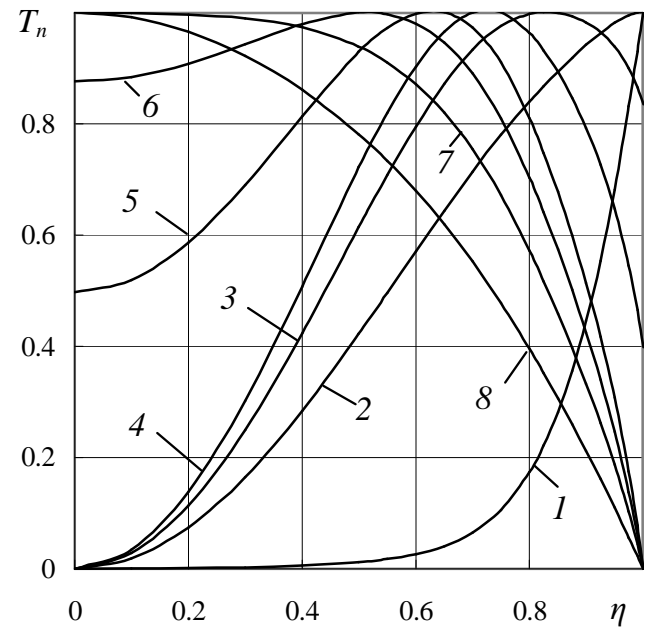

Fig. 6 Regularities of temperature field change in evaporating water droplets in the case of combine heating. Fo: (1) 0.0176, (2) 0.267 , (3) 0.332 , (4) 0.354 , (5) 0.375 , (6) 0.397 , (7) 0.429, (8) 2.403. $R_{0}=0.0001 \mathrm{~m} . T_{g}=1500 \mathrm{~K} . T_{l, 0}=300 \mathrm{~K} . p_{v, \infty} / p=0$.

In a primary water droplet heating period $T_{n} \equiv 1$ is on the droplet surface while $T_{n} \equiv 0$ is in the center (Fig. 6 curve 1). Transitional heating period starts when $T_{n} \equiv 1$ retires from the droplet surface. According to the peculiarities of $T_{n}$ during the transitional droplet heating period, first, second and the third part may be pointed. In the first part of transitional heating $T_{n} \equiv 1$ is inside the droplet while $T_{n} \equiv 0$ is in the droplet center (see Fig. 6 curves 2-4). In the second part of transitional heating $T_{n} \equiv 1$ is inside the droplet while $T_{n} \equiv 0$ is in the droplet surface (see Fig. 6 curves 5, 6). The first and second periods are connected by a moment in which $T_{R}=T_{C}$. These temperatures meet the condition $T_{n} \equiv 0$. In the third part of transitional heating $T_{n} \equiv 1$ is in a droplet center while $T_{n} \equiv 0$ is on its surface. This period begins when $T_{n} \equiv 1$ moves to the droplet center. It continues till the commencing of droplet equilibrium evaporation. The commencing of the final droplet heating stage can not be determined according to the peculiarities of $T_{n}$ because also in the final droplet heating stage $T_{n} \equiv 1$ is in the droplet center. This requires additionally taking into account that during equilibrium evaporation energy supplied on a droplet does not heat it. Therefore in the final heating stage all energy supplied on the droplet evaporates it. In above mentioned evaporating droplet heating stages the conditions for complex interaction of transfer processes are different. This is determined by the peculiarities of energy flows densities changes on the droplet surface (see Fig. 7)

During unsteady evaporation a droplet heats, therefore evaporation process is supported by only a part of the energy taken on it. Other part heats the droplet $q_{\Sigma}^{+}(\tau) \equiv q_{r}^{+}(\tau)+q_{c}^{+}(\tau)=q_{f}^{+}(\tau)+q_{h}(\tau)$. Intensity of the energy employed for droplet heating is outlined by the total heat flow density on internal side of the droplet surface $q_{h}(\tau) \equiv q_{\Sigma}^{-}(\tau)$ and $q_{f}^{+}(\tau)=q_{\Sigma}^{+}(\tau)-q_{\Sigma}^{-}(\tau)$. Energy flow densities on an interphase contact surface are determined by the interrelation of the transfer processes interaction in a droplet and in its ambience. In this case convective component $q_{c}^{-}(\tau)$ of total heat flow $q_{\Sigma}^{-}(\tau)$ density is determinant. It undergoes not only quantitative but also and essential qualitative changes during the droplet heating and evaporation. Temperature field gradient in a droplet is positive in the primary heating period. Therefore the droplet is heated by the absorbed radiative energy and a part of convective heat flow $q_{h}(\tau)=q_{r}^{-}(\tau)+k_{c} q_{c}^{+}(\tau)$. Other convective heat flow part evaporates the droplet $q_{f}^{+}(\tau)=\left(1-k_{c}\right) q_{c}^{+}(\tau)$. At the beginning of the heating $k_{c}$ is close to 1 . It reduces to zero during the primary period. So during primary droplet heating $q_{c}^{-}(\tau)$ corresponds to the 
external convective heat flow part which heats the droplet. Its vector is directed to the droplet center, so $q_{\Sigma}^{-}(\tau) \equiv q_{r}^{-}(\tau)+q_{c}^{-}(\tau)$. Temperature gradient in a droplet is negative during the transitional heating period. The droplet is heated by a part of absorbed radiating energy $q_{h}(\tau)=k_{r} q_{r}^{-}(\tau)$. It is evaporated by all convective heating energy and the rest of the radiation flow $q_{f}^{+}(\tau)=q_{c}^{+}(\tau)+\left(1-k_{r}\right) q_{r}^{-}(\tau)$. Coefficient $k_{r}$ decreases from one to zero during the transitional heating period. At the end of the transitional period and later $k_{r} \equiv 0$. So during a droplet transitional heating period $q_{c}^{-}(\tau)$ corresponds to the part of absorbed by the droplet radiating flow density which evaporates the droplet. Its vector is directed to the droplet surface $q_{\Sigma}^{-}(\tau) \equiv q_{r}^{-}(\tau)-q_{c}^{-}(\tau)$.

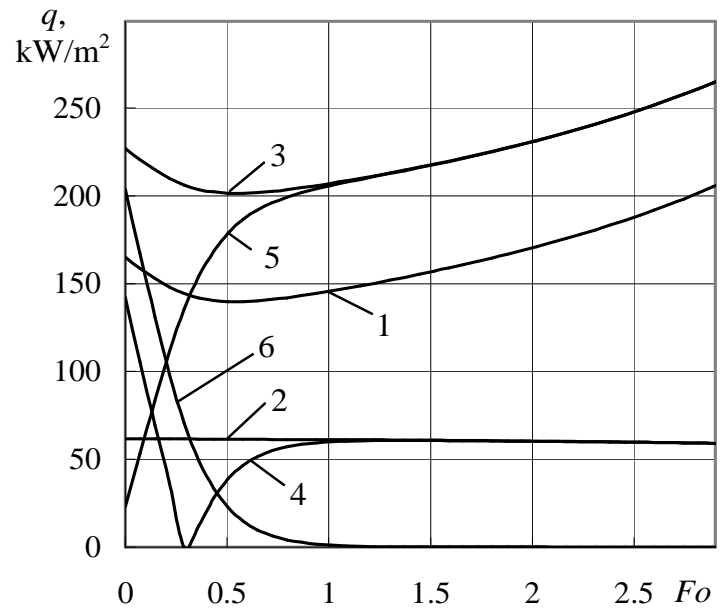

Fig. 7 Regularities of the changes of energy flow densities on the surfaces of evaporating water droplet. Combine water droplet heating case, when $\Delta w \equiv 0 . \quad R_{0}=0,0002 \mathrm{~m} . \quad T_{l, 0}=300 \mathrm{~K}$. $T_{g}=1073 \mathrm{~K} . p_{v, \infty} / p=0$. Meaning of a parameter $q$ : (1) $q \equiv q_{c}^{+}$, (2) $q \equiv q_{r}^{+}$, (3) $q \equiv q_{\Sigma}^{+}$, (4) $q \equiv q_{c}^{-}$, (5) $q \equiv q_{f}^{+}$, (5) $q \equiv q_{\Sigma}^{-}$.

\section{CONCLUSIONS}

The droplet heating manner is essential factor determining the peculiarities of sprayed liquid thermal state change. The important factors are: liquid spray dispersity - determines droplet vaporization velocity and their expansion in the case of heating; initial liquid temperature - makes preconditions for the phase transformation by condensation regime; gas flux parameters - determine the impact of external energy intensity on droplets. Thermal state change of evaporating droplet may be classified by instantaneous temperature field peak position in the droplet, accentuating primary, transitive and final droplet heating periods.

The research results show that irrespective of droplet dispersivity a peculiar change of sprayed liquid thermal state exists in the time scale expressed by Fourier number. The above mentioned change can be conveniently defined by the characteristic curves representing the change of a droplet surface, centre and bulk temperatures, which are sensibly influenced by temperature of gas mixture and partial pressure of liquid vapour in it. The density of phase transformations heat flow:

The research revealed influence of droplet heating mode to their thermal state. The characteristic lifetime of the conductively heated droplet in the scale, expressed by Fourier number: $0 \div F o_{c o, k} \div F o_{e, k} \div F o_{f, k}$, independent of sprayed water dispersity.

During the condensation mode of phase transformation $0 \div F o_{c o, k}$ the droplet is heated by condensing water vapor together with conductive heat from air. The time instant $F o \equiv F o_{c o, k}$ reflects the characteristic duration of the mode of condensation phase transformations, which is determined by temperature and humidity of air and temperature of sprayed water into the evaporation chamber. During the condensation mode $q_{f}^{+}\left(F o \rightarrow F o_{c o, k}\right) \rightarrow 0$ the droplet warms up till reaches dew point. Time instance, when nature of phase transformation at droplet surface changes is $q_{k}^{-}\left(F o_{c o, k}\right) \equiv q_{k}^{+}\left(F o_{c o, k}\right)$. During the condensation mode it is possible to dehumidify an air. During the unsteady evaporation mode $F o_{c o, k} \div F o_{e, k}$ heat supplied to the droplet by conduction heats them and causes its evaporation. As warm-up of the droplet declines and evaporation increases, the process gradually reaches equilibrium evaporation mode, which by definition onsets when entire heat supplied to the droplet is consumed for evaporation of the droplet. At time instance $F o \equiv F o_{e, k}$ droplets warm-up till temperature $T_{e, k}$, this identifies the mode of equilibrium evaporation. During the equilibrium evaporation mode the temperature $T_{e, k}$ remains constant.

\section{NOMENCLATURE}

$\begin{array}{ll}a & \text { thermal diffusivity }\left(\mathrm{m}^{2} / \mathrm{s}\right) \\ B_{T} & \text { Spalding transfer number } \\ c_{p} & \text { specific heat }(\mathrm{J} / \mathrm{kg} \cdot \mathrm{K}) \\ F O & \text { Fourier number } \\ I & \text { index of control time } \\ J & \text { index of droplet surface } \\ L & \text { latent heat of evaporation }(\mathrm{J} / \mathrm{kg})) \\ m_{v} & \text { vapour mass flux density }\left(\mathrm{kg} / \mathrm{m}^{2} \mathrm{~s}\right) \\ n & \text { number of the term in infinite sum } \\ N u & \text { Nusselt number } \\ p & \text { pressure }(\mathrm{Pa}) \text { time }(\mathrm{s}) \\ P e & \text { Peclet number } \\ q & \text { heat flux density }\left(\mathrm{W} / \mathrm{m}^{2}\right) \\ r & \text { coordinate of a droplet }(\mathrm{m}) \\ R & \text { radius of a droplet }(\mathrm{m}) \\ R_{\mu} & \text { universal gas constant }(\mathrm{J} / \mathrm{kmol} \mathrm{K}) \\ t & \text { control time }(\mathrm{s}) \\ T & \text { temperature }(\mathrm{K})\end{array}$

\section{Greek Symbols}

$\Delta w \quad$ droplet slip velocity in the gas $(\mathrm{m} / \mathrm{s})$

$\eta \quad$ non-dimensional droplet coordinate

$\lambda$ thermal conductivity $(\mathrm{W} / \mathrm{m} \mathrm{K})$

$\mu \quad$ molecular mass $(\mathrm{kg} / \mathrm{kmol})$

$\rho \quad$ density $\left(\mathrm{kg} / \mathrm{m}^{3}\right)$

$\tau \quad$ time (s)

\section{Superscripts}

$+\quad$ external side of a droplet surface

- $\quad$ internal side of a droplet surface

Subscript

c convective

co condensation regime

$e \quad$ equilibrium evaporate regime

ef effective

$f \quad$ phase transformation

g gas

$i \quad$ time index in a digital scheme

I index of control time

$I_{\omega} \quad$ intensity of spectral radiation

$j \quad$ index of droplet cross-section

$J \quad$ index of droplet surface

$k \quad$ conductive

$l \quad$ liquid 


$\begin{array}{ll}m & \text { mass average } \\ r & \text { radiative } \\ R & \text { droplet surface } \\ v & \text { vapour } \\ v g & \text { vapour-gas mixture } \\ 0 & \text { initial state } \\ \Sigma & \text { total } \\ \infty & \text { far from a droplet }\end{array}$

\section{REFERENCES}

Miliauskas, G., Sabanas, V., 2006, "Interaction of transfer processes during unsteady evaporation of water droplets," International Journal of Heat and Mass Transfer, 49, 1790-1803. http://dx.doi.org/10.1016/j.ijheatmasstransfer.2005.11.015

Tseng, C.C., Viskanta, R., 2006, "Enhancement of water droplet evaporation by radiation absorption," Fire Safety J., 41, 236-247. http://dx.doi.org/10.1016/j.firesaf.2006.01.001

Sazhin, S.S., 2006, "Advanced models of fuel droplet heating and evaporation," Progress in Energy and Combustion Science, 32, 162214.

http://dx.doi.org/10.1016/j.pecs.2005.11.001

Miliauskas, G., Sinkunas, S., Miliauskas G., 2010, "Evaporation and condensing augmentation of water droplets in flue gas," International Journal of Heat and Mass Transfer, 53, 1220-1230. http://dx.doi.org/10.1016/j.ijheatmasstransfer.2009.10.005

Shorin, S., 1964, Teploperedacha, Moskva, Visshaja Shkola.
Miliauskas, G., 2001, "Regularities of unsteady radiative-conductive heat transfer in evaporating liquid droplets," International Journal of Heat and Mass Transfer, 44, 785-798. http://dx.doi.org/10.1016/S0017-9310(00)00127-7

Abramzon, B., Sirignano W.A., 1989, "Droplet vaporization model for spray combustion calculations," International Journal of Heat and Mass Transfer, 32, 1605-1618. http://dx.doi.org/10.1016/0017-9310(89)90043-4

Kuzikovskij, A.V., 1973, "Dynamic of spherical particle in powerful optical field,” Izv. VUZ Fizika, 5, 89-94.

Hale, G.M., Querry, M.R., 1973, "Optical constants of water in the 200-nm to 200- $\mu \mathrm{m}$ wavelength region," Appl. Optics, 12, 555-562. http://dx.doi.org/10.1364/AO.12.000555

Hale, G.M., Querry, M.R., Rusk, A.N., Williams, D., 1972, "Influence of temperature on the spectrum of water," J. Opt. Soc. Am. , 62, 11031108.

http://dx.doi.org/10.1364/JOSA.62.001103

Miliauskas, G., Garmus, V., 2004, "Peculiarities of heated liquid evaporating droplets state change," Mechanics, 5, 31-38.

Miliauskas, G., $\quad$ Sabanas, V., $\quad$ Bankauskas, R., $\quad$ Miliauskas, G., Sankauskaite, V., 2008, "The peculiarities of sprayed liquid's thermal state change, as droplets are heated by conduction," International Journal of Heat and Mass Transfer, 51, 4145-4160. http://dx.doi.org/10.1016/j.ijheatmasstransfer.2008.01.016 